\title{
Distribuição da carga interna de treinamento durante uma temporada no voleibol profissional
}

http://dx.doi.org/10.11606/1807-5509202000040567

\author{
Heglison Custódio Toledo* \\ Bernardo Miloski* \\ Yuri de Almeida Costa Campos ${ }^{* * *}$ \\ Rosa Virginia Diaz Guerrero*/**** \\ Renato Miranda* \\ J eferson Macedo Vianna* \\ Maurício Bara Filho*
}

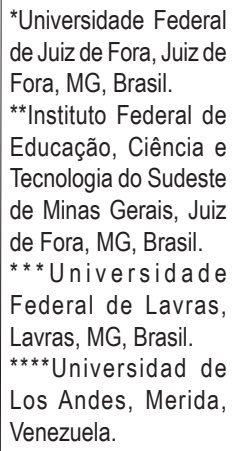

*Universidade Federal de Juiz de Fora, Juiz de Fora, MG, Brasil. ${ }^{* *}$ Instituto Federal de Educação, Ciência e Tecnologia do Sudeste de Minas Gerais, Juiz de Fora, MG, Brasil.

*** Universidade Federal de Lavras, Lavras, MG, Brasil. ****Universidad de Los Andes, Merida, Venezuela.

0 objetivo do presente estudo foi descrever a distribuição da carga interna de treinamento (CIT) direcionada a diferentes tipos de treinamentos durante uma temporada de jogadores de voleibol profissionais. A CIT foi registrada em todas as sessões realizadas durante 30 semanas de treinamento da temporada, considerando os seguintes tipos de treinamento: força $(F)$, técnico $(T)$, técnico-tático $(T)$, jogo $(J)$ e condicionante + técnico-tático $(\mathrm{C}+\mathrm{T})$. A temporada foi dividida em 4 periodos: preparatório $(\mathrm{PP})$ com 8 semanas, competitivo I (PCI) com 6 semanas, competitivo II (PCII) com 7 semanas, e competitivo III (PCIII) com 9 semanas. Para quantificação da CIT foi utilizado o método PSE-sessão. Os resultados apontam uma alternância na magnitude da $\mathrm{CIT}$ durante as semanas da temporada; a redução da $\mathrm{CIT}$ direcionada a $\mathrm{F}$ de $\mathrm{PCI}$ para $\mathrm{PCII}(\mathrm{p}$ $=0,04)$; maiores valores de CIT para $T$ em PCl e PCIII comparados a PP $(p<0,01)$; e menores valores de CIT para $\mathrm{C}+\Pi$ em $\mathrm{PCll}$ comparado aos demais períodos $(\mathrm{p}<0,01)$. A partir dos resultados do presente estudo pode-se concluir que a $\mathrm{CIT}$ apresentou uma distribuição que alternou diferentes magnitudes durante as semanas de treinamento na temporada, que ocorre uma ênfase na CIT direcionada aos atributos técnicotáticos e redução da CIT destinada ao desenvolvimento da aptidão física durante o período competitivo da temporada do voleibol.

Palavras-chave: PSE-sessão; Periodização; Monitoramento; Carga de treinamento.

\section{Introdução}

A organização do processo de treinamento nos esportes coletivos busca gerar condiçôes para que o atleta sustente um nível ótimo de desempenho durante o período de competiçóes ${ }^{1}$. Baseado nessa premissa, jogadores de voleibol cumprem rotinas com diferentes tipos de treinamento para o desenvolvimento de atributos físicos, técnicos e táticos necessários ao melhor desempenho nos jogos durante a temporada ${ }^{2-3}$. Essa melhora do desempenho esportivo, por sua vez, dependerá da distribuição planejada e estruturada das cargas de treinamento, de forma a proporcionar um equilíbrio entre as demandas de estresse e os períodos de recuperação ${ }^{1}$. Esse equilíbrio seria um fator importante para minimizar possíveis quedas no rendimento esportivo, e proporcionar a manutenção da excelência física, técnica e tática ao longo da temporada.

Com o intuito de explicar essa relação dose-resposta referente ao processo de treinamento, IMPELLIZERI et al. ${ }^{4}$ propuseram que as adaptaçóes induzidas pelo treinamento são desencadeadas pelo estresse psicofisiológico que é imposto ao organismo do atleta, que representa a carga interna de treinamento (CIT). Uma vez que as respostas adaptativas decorrentes do treinamento estão ligadas a CIT aplicada ${ }^{4}$, nota-se a importância de treinadores e demais envolvidos na área compreenderem como essa variável está distribuída ao 
longo da temporada esportiva, tanto no que diz respeito a magnitude (cargas baixas, moderadas e altas), quanto no que se refere a seus objetivos (ex. treinamentos físicos, técnicos e táticos).

Embora a necessidade de se compreender a distribuição das cargas de treinamento suscite a realização de investigaçóes acerca do tema, até pouco tempo, observava-se uma dificuldade em se quantificar a CIT utilizando-se uma unidade de medida comum aos diferentes tipos de treinamento presentes na rotina dos esportes coletivos. Nesse contexto, o método da percepção subjetiva do esforço da sessão (PSE-sessão) proposto por Foster et al. ${ }^{5}$, se apresentou como uma ferramenta simples, válida e fidedigna para quantificar a CIT em diferentes esportes coletivos, tais quais rúgbi ${ }^{6}$, futebol ${ }^{7-4} \mathrm{evoleibol}^{8}$. Segundo a proposta de FosTER et al. ${ }^{5}$ a CIT é obtida a partir do produto entre o escore de percepçáo de esforço reportado pelo atleta referente a sessão de treinamento e o tempo total da sessão em minutos. Assim, uma das vantagens em se utilizar o método está na sua praticidade para quantificar a CIT de diferentes tipos, como por exemplo, força, resistência, técnica e tática, possibilitando a construção de gráficos específicos que permitem a visualização da sua dinâmica durante um determinado período de treinamento.

No que diz respeito a distribuição das cargas de treinamento no voleibol, Freitas, Miloski e BaraFILHO $^{2}$ recentemente reportaram uma dinâmica com incremento da CIT do primeiro para o segundo mesociclo, e posterior manutençáo da mesma no restante da temporada de uma equipe adulta

\section{Metódo}

Quinze jogadores profissionais de uma equipe de voleibol que disputava a Superliga Masculina 2015-2016, principal competição da modalidade no Brasil, participaram voluntariamente do estudo. Os jogadores treinavam entre 4 a 6 dias por semana, cumprindo de 1 a 2 sessóes por dia. Todos jogadores apresentavam no mínimo 3 anos de experiência em treinamento voltado para competiçôes de nível nacional. Os jogadores que participaram de menos de $75 \%$ do número total sessóes de treinamento realizados na temporada tiveram seus dados excluídos da análise. Dessa forma, participante da divisão de acesso à Superliga no Brasil. Em outro estudo realizado no voleibol brasileiro, AокI et al. ${ }^{9}$ mostraram resultados diferentes, com maiores valores de CIT na etapa preparatória em relação a competitiva em jovens jogadores. Esse estudo ainda demonstrou que durante a etapa preparatória, jogadores da categoria sub-19 apresentam ênfase na CIT dedicada ao desenvolvimento da força, ao passo que na categoria sub-16 a CIT está mais direcionada a treinamentos que conjugam diferentes capacidades físicas na mesma sessão. Embora esses estudos apresentem possibilidades para a distribuição da CIT em jogadores jovens e adultos, a maneira como a CIT é distribuída em cada tipo de treinamento (técnico, tático e físico) durante a temporada de equipes da divisão principal do voleibol brasileiro ainda é desconhecida. Estudos que apresentem esse tipo de distribuição podem fornecer informaçōes relevantes para orientar profissionais da área na elaboração de seus planejamentos, e ainda, para promover futuras discussóes acerca das formas mais utilizadas de distribuiçáo da CIT. Dessa maneira, o objetivo do presente estudo foi descrever a distribuição da CIT direcionada a diferentes tipos de treinamento durante uma temporada de jogadores de voleibol profissionais brasileiros. Tem-se a hipótese que a CIT apresentará valores mais elevados no período de preparação em comparação ao período competitivo, em virtude de maiores valores de CIT destinados ao desenvolvimento da aptidáo física no período de preparação.

foram analisados os dados referentes a 7 jogadores (idade 26,6 \pm 4,7 anos; massa corporal 91,5 \pm 8,5 $\mathrm{kg}$; estatura 194,1 $\pm 6,0$; IMC $22,0 \pm 7,5 \mathrm{~kg} / \mathrm{m}^{2}$ ) que cumpriram tal critério. Os procedimentos do estudo respeitaram as normas internacionais de experimentação com humanos em conformidade com a última Declaraçáo de Helsinque, sendo o projeto submetido e posteriormente aprovado pelo Comitê de Ética com Pesquisa em Humanos da Universidade Federal de Juiz de Fora sob o parecer no 278/2010. Os jogadores assinaram um termo de consentimento autorizando a coleta e a divulgaçáo dos dados. 


\section{Delineamento experimental}

A CIT foi registrada em todas as sessóes realizadas durante as 30 semanas de treinamento da temporada. Todos os treinamentos foram planejados e conduzidos pela comissáo técnica da equipe analisada. A temporada foi dividida em 4 períodos: preparatório (PP) com 8 semanas, no qual a equipe participou jogos amistosos preparatórios e da primeira fase do campeonato estadual; competitivo I (PCI) com 6 semanas, no qual foram disputados na seguinte sequência a fase final do campeonato estadual, dois jogos do primeiro turno da Superliga Nacional e a fase final dos jogos abertos do estado; competitivo II (PCII) com 7 semanas, no qual foram disputados nove jogos do primeiro turno da Superliga Nacional e finalizado com uma semana de interrupçáo da competiçáa em virtude do recesso de fim de ano; e competitivo III (PCIII) com 9 semanas, no qual foi disputado o segundo turno da Superliga Nacional. Para quantificação da CIT foi utilizado o método PSE-sessão (21), obtida nos seguintes tipos de treinamento utilizados:

i) Força (F): sessōes de treinamento com pesos, pliometria e lançamentos de medicine ball realizados na sala de musculação.

ii) Técnico (T): sessóes de treinamento para desenvolvimento dos fundamentos saque, passe, levantamento, bloqueio e defesa fora do formato de jogo.

iii) Técnico-tático (TT): sessóes de treinamento que utilizavam formato de jogo com regras adaptadas para desenvolvimento dos fundamentos técnicos e táticos.

iv) Jogo (J): CIT registrada nos amistosos e jogos oficiais.

v) Condicionante + Técnico tático $(\mathrm{C}+\mathrm{TT})$ : sessóes de treinamento nas quais eram realizados exercícios de deslocamento para desenvolvimento de atributos da aptidáo física (resistência e velocidade), seguido posteriormente por exercícios característicos da sessão técnico-tática.

O número de sessôes e a duração média das mesmas em cada período de treinamento é apresentado na TABELA 1. A TABELA 2 apresenta os objetivos principais das semanas de treinamento distribuídas em cada período.

Os jogadores tinham acesso a orientaçáo nutricional de um profissional especializado, que prescrevia os planos de alimentaçáo e hidrataçáo de acordo com as necessidades individuais. Adicionalmente, os jogadores faziam uso de medicamentos (ex. anti-inflamatórios, relaxantes musculares) durante a temporada quando o staff médico julgava necessário.

TABLE 1 - Média de duração e número de sessões de treinamento durante os períodos da temporada.

\begin{tabular}{|c|c|c|c|c|c|c|c|c|c|}
\hline & \multicolumn{2}{|c|}{ PP } & \multicolumn{2}{|c|}{ PCI } & \multicolumn{2}{|c|}{ PCII } & \multicolumn{2}{|c|}{ PCIII } & \multirow{7}{*}{$\begin{array}{l}\mathrm{PP}=\text { período } \\
\text { preparatório; } \\
\mathrm{PCl}=\text { período } \\
\text { competitivo I; } \\
\text { PCII = período } \\
\text { competitivo II; } \\
\text { PCIII = período } \\
\text { competitivo III; } \\
\mathrm{F}=\text { força; } \\
\mathrm{J}=\text { jogo; } \\
\mathrm{T}=\text { técnico; } \\
\mathrm{TT}=\text { técnico-tático; } \\
\mathrm{C}+\mathrm{TT}=\text { condicionante } \\
\text { e técnico-tático. }\end{array}$} \\
\hline & $\begin{array}{l}\text { No de } \\
\text { sessōes }\end{array}$ & $\begin{array}{l}\text { Duração sessão } \\
\quad(\min )\end{array}$ & $\begin{array}{l}\text { No de } \\
\text { sessóes }\end{array}$ & $\begin{array}{l}\text { Duração sessão } \\
\qquad(\min )\end{array}$ & $\begin{array}{l}\text { No de } \\
\text { sessóes }\end{array}$ & $\begin{array}{c}\text { Duração } \\
\text { sessão (min) }\end{array}$ & $\begin{array}{l}\text { No de } \\
\text { sessóes }\end{array}$ & $\begin{array}{c}\text { Duração sessão } \\
\text { (min) }\end{array}$ & \\
\hline $\mathrm{F}$ & 26 & $58,46 \pm 5,32$ & 22 & $60 \pm 0$ & 17 & $60 \pm 0$ & 30 & $60 \pm 0$ & \\
\hline $\mathrm{J}$ & 14 & $98,92 \pm 25,92$ & 8 & $99,37 \pm 23,24$ & 9 & $\begin{array}{c}123,33 \pm \\
22,11\end{array}$ & 11 & $122,72 \pm 23,77$ & \\
\hline $\mathrm{T}$ & 22 & $38,95 \pm 15,64$ & 8 & $31,87 \pm 17,12$ & 28 & $\begin{array}{c}31,67 \pm \\
12,63\end{array}$ & 28 & $31,96 \pm 18,14$ & \\
\hline TT & 18 & $50,72 \pm 9,83$ & 18 & $48,88 \pm 12,64$ & 22 & $\begin{array}{c}51,81 \pm \\
14,10\end{array}$ & 31 & $51,67 \pm 13,30$ & \\
\hline $\mathrm{C}+\mathrm{TT}$ & 15 & $71,73 \pm 25,56$ & 11 & $74,54 \pm 23,68$ & 3 & $68,33 \pm 8,49$ & 14 & $61,07 \pm 18,53$ & \\
\hline
\end{tabular}


TABLE 2 - Objetivos principais das semanas distribuídas nos períodos de treinamento.

FM = força máxima; TT = técnico-tático; $\mathrm{V} / \mathrm{P}=$ velocidade $\mathrm{e}$ potência;

$\mathrm{R}=$ recuperação.

\begin{tabular}{|c|c|c|c|c|c|c|c|c|c|c|c|c|c|c|c|c|}
\hline \multirow{2}{*}{$\begin{array}{l}\text { Período } \\
\text { Semana }\end{array}$} & \multicolumn{5}{|c|}{ Período preparatório } & \multicolumn{4}{|c|}{ Período competitivo I } & \multicolumn{3}{|c|}{ Período competitivo II } & \multicolumn{4}{|c|}{ Período competitivo III } \\
\hline & $1-2$ & 3 & $4-5$ & 6 & $7-8$ & $9-10$ & 11 & $12-13$ & 14 & $15-17$ & $18-20$ & 21 & $22-23$ & $24-27$ & $28-29$ & 30 \\
\hline \multirow[t]{2}{*}{ Objetivos } & FM & $\mathrm{V} / \mathrm{P}$ & FM & $\mathrm{V} / \mathrm{P}$ & $\mathrm{R}$ & FM & $\mathrm{V} / \mathrm{P}$ & FM & $\mathrm{V} / \mathrm{P}$ & $\mathrm{V} / \mathrm{P}$ & FM & $\mathrm{R}$ & FM & $\mathrm{V} / \mathrm{P}$ & FM & $\mathrm{R}$ \\
\hline & TT & TT & $\mathrm{TT}$ & TT & & $\mathrm{TT}$ & TT & TT & TT & TT & TT & & TT & TT & TT & \\
\hline
\end{tabular}

\section{Carga interna de treinamento}

A CIT foi registrada utilizando-se o método PSE-sessão. O escore de PSE-sessão foi registrado 30 minutos após o término da sessão, de acordo com a orientação de estudos anteriores ${ }^{4-10}$, utilizando-se a escala de Borg adaptada (CR-10) por Foster et al. 5 . Sem que houvesse contato entre os atletas, cada jogador apontava sua resposta na escala referente a pergunta: "Como foi o seu treino?", considerando a sessão de treinamento como um todo. A CIT foi obtida a partir do produto entre o escore de PSE-sessáo reportado pelo atleta ao final da sessão e o tempo total de treinamento em minutos. Todos jogadores eram familiarizados com o instrumento. O somatório da CIT semanal (sCIT) em cada tipo de treinamento (F, T, TT, J e C+TT) e a média do somatório da CIT semanal (média-sCIT) aplicada em cada período de treinamento (PP, PCI, PCII e PCIII) foram retidos para análise. Adicionalmente, a sCIT foi categorizada em baixa, moderada-baixa,

\section{Resultados}

A FIGURA 1 ilustra a distribuição da sCIT de acordo com a magnitude (baixa, moderada-baixa, moderada-alta e alta) e tipo de treinamento (F, T, TT, J e C+TT) durante as semanas de treinamento.

Verificou-se diferença significante para médiasCIT total entre os períodos de treinamento $(\mathrm{F}=$ $9,1 ; \mathrm{gl}=3 ; p<0,01)$. Foram observados menores valores de média-sCIT total em PCII comparado a PCI e PCIII $(p<0,01)$.

A ANOVA two-way demonstrou significância para os fatores tipo de treinamento $(\mathrm{F}=62,0 ; p<$ $0,01)$ e períodos de treinamento $(\mathrm{F}=7,7 ; \mathrm{gl}=8$; $p<0,01)$. Não foram observadas diferenças entre média-sCIT implementada nos diferentes tipos de moderada-alta e alta de acordo com orientaçóes de Miloski et al. ${ }^{11}$.

\section{Análise estatística}

Para verificar a normalidade e a homogeneidade das variâncias foram adotados os testes de Shapiro Wilk e Levene, respectivamente. A esfericidade dos dados foi avaliada por meio do teste $\mathrm{M}$ de Box, e caso a esfericidade fosse violada, foi utilizado o fator de correção Épsilon de Huynh-Feldt. Atendidos os pressupostos de normalidade e homogeneidade de variâncias, foram aplicados: o teste de ANOVA one-way de medidas repetidas seguido pelo post hoc de Tukey para comparar a média-sCIT entre os períodos; e o teste ANOVA two-way seguido pelo post hoc de Tukey para comparar a média-sCIT entre os fatores tipo (F, T, TT, $\mathrm{J}$ e C+TT) e período de treinamento (PP, PCI, PCII e PCIII). Foi adotado nível de significância $p \leq 0,05$. O tratamento estatístico foi realizado no software SPSS (20.0, IBM, Armonk, USA). Os dados são reportados em média e desvio-padrão. treinamento em PP. Em PCI, foram observados maiores valores de média-sCIT para $\mathrm{F}$ em relação a J $(p<0,01)$ e T $(p=0,02)$; e para TT em relação aos demais tipos de treinamento $(p>0,01)$. Durante PCII, a análise identificou menores valores de média-sCIT para T e C+TT em comparação a $\mathrm{F}(p$ $\leq 0,01)$, J e TT $(p<0,01)$; e maiores valores para TT em comparação a $\mathrm{F}(p<0,01)$ e J $(p=0,04)$. Em PCIII, foram observados maiores valores de média-sCIT para TT em relaçáo aos demais tipos de treinamento $(p<0,01)$.

Além disso também foram observados: redução da média-sCIT direcionada a F de PCI para PCII $(\mathrm{p}=0,04)$; maiores valores de média-sCIT para TT 
em PCI e PCIII comparados a PP $(\mathrm{p}<0,01)$; e menores valores de média-sCIT para $\mathrm{C}+\mathrm{TT}$ em PCII comparado aos demais períodos $(p<$
0,001). Os valores de média-sCIT de cada tipo de treinamento durante os períodos da temporada estão expostos na TABELA 2.

FIGURA 2 - Somatório da carga interna de treinamento semanal durante a temporada.

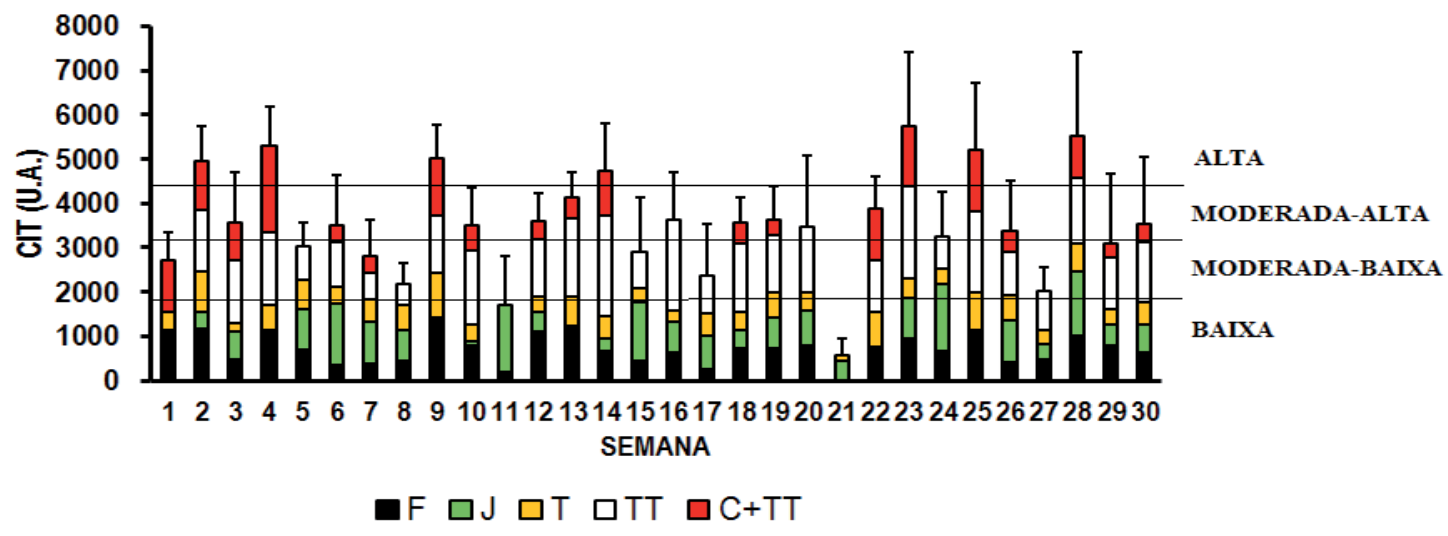

sCIT = somatório carga interna de treinamento semanal; U.A. = unidades arbitrárias;

$F=$ força;

$\mathrm{J}=$ jogo;

$\mathrm{T}=$ técnico;

TT = técnico-tático;

$\mathrm{C}+\mathrm{TT}=$ condicionante

+ técnico tático.

TABLE 3 - Média do somatório de carga interna semanal decada tipo de treinamento nos períodos da temporada.

\begin{tabular}{lcccc}
\hline & PP & PCI & PCII & PCIII \\
\hline F & $739,3 \pm 83,9$ & $894,3 \pm 154,3^{\mathrm{d}}$ & $519,8 \pm 97,9^{2, \mathrm{c}, \mathrm{d}, \mathrm{e}}$ & $769,0 \pm 105,0^{\mathrm{d}}$ \\
$\mathrm{J}$ & $633,0 \pm 289,7$ & $455,8 \pm 188,2^{\mathrm{a}, \mathrm{d}}$ & $824,7 \pm 340,8^{\mathrm{c}, \mathrm{d}, \mathrm{e}}$ & $741,5 \pm 261,6^{\mathrm{d}}$ \\
T & $550,3 \pm 43,8$ & $494,4 \pm 59,4^{\mathrm{a}, \mathrm{d}}$ & $390,2 \pm 74,3$ & $534,0 \pm 65,5^{\mathrm{d}}$ \\
TT & $910,3 \pm 131,1$ & $1384,9 \pm 205,1^{1}$ & $1199,5 \pm 291,3^{\mathrm{c}, \mathrm{e}}$ & $1353,5 \pm 397,4^{1}$ \\
C+TT & $745,1 \pm 64,5^{3}$ & $671,0 \pm 70,4^{3, \mathrm{~d}}$ & $117,5 \pm 14,7$ & $678,7 \pm 225,4^{3, \mathrm{~d}}$ \\
CIT TOTAL & $3578,0 \pm 409,6$ & $3900,4 \pm 463,0^{3}$ & $3051,7 \pm 664,4$ & $4076,6 \pm 881,8^{3}$ \\
\hline
\end{tabular}

\section{Discussão}

PP = período preparatório; $\mathrm{PCl}=$ período competitivo I; $\mathrm{PCI}=$ período competitivo II; PCIII = período competitivo III; $F=$ força;

$\mathrm{J}=$ jogo;

$\mathrm{T}=$ técnico;

$\mathrm{TT}=$ técnico-tático;

$\mathrm{C}+\mathrm{TT}=$ condicionante e técnico-tático.

1, 2, 3 e 4 = diferença significante para $\mathrm{PP}$,

$\mathrm{PCl}$, PCll e PCIII, respectivamente; $a, b, c, d, e=$ diferença significante para F, J, T, TT e $\mathrm{C}+\mathrm{TT}$, respectivamente ( $p$ $\leq 0,05$ ).
Os principais achados do presente estudo foram: i) a CIT semanal apresentou grande alternância de magnitude no decorrer de toda temporada; ii) ocorreu uma redução da magnitude da CIT no segundo momento de período competitivo (PCII); iii) o segundo momento de período competitivo também apresentou valores mais baixos de CIT direcionada a F e C+TT; iv) ocorreu um aumento da magnitude da CIT TT no período de competições comparada ao período preparatório; v) durante o período de competiçóes ocorreu uma ênfase na CIT direcionada ao desenvolvimento TT, em comparação aos outros conteúdos.

Em primeiro lugar, nota-se que a CIT total semanal apresentou grande alternância entre valores de diferentes magnitudes (baixa, moderada-baixa, moderada-alta e alta) durante toda a temporada, tanto para o período preparatório como para o 
competitivo, o que corrobora os resultados de Freitas, Miloski e Bara-Filho ${ }^{2}$ em jogadores da segunda divisão do voleibol brasileiro. Essa alternância na magnitude da CIT ao longo das semanas de treinamento também foi reportada durante o período competitivo de jogadores de futsal brasileiros profissionais ${ }^{11-12}$. Entretanto, pode-se observar que, diferentemente do presente estudo, os jogadores de futsal foram submetidos a sucessivas semanas com cargas elevadas durante o período preparatório ${ }^{11-12}$. A maior alternância na magnitude da CIT observada durante o período preparatório da presente investigação pode ser explicada, possivelmente, pela maior incidência de jogos da equipe analisada. $\mathrm{O}$ maior número de jogos oficiais pode ter representado uma demanda de estresse mais elevada comparada aos estímulos de treinamento ${ }^{13-14}$ e dificultado o processo de recuperação em virtude de fatores como viagens e mudança na rotina alimentar e de sono ${ }^{15}$, levando os treinadores a planejarem uma alternância maior na magnitude das cargas de treinamento nesse período em busca de minimizar a aparição de adaptaçóes indesejadas. Esses resultados em conjunto apontam para um padrão de alternância na distribuição da magnitude da CIT em esportes coletivos, que estaria ligado as necessidades impostas pelo calendário competitivo. Nesse caso, a aplicaçáo de CIT semanal com valores mais elevados buscariam o incremento da aptidáo dos atletas ${ }^{11}$, enquanto as semanas com CIT mais baixas buscam evitar uma demanda de estresse excessiva (decorrente da alta intensidade dos treinamentos e jogos, viagens longas que podem comprometer o sono dos atletas, entre outros fatores), com intuito de que o atleta consiga estar recuperado física e mentalmente para o próximo compromisso competitivo ${ }^{16}$.

Adicionalmente, o presente estudo também demonstrou menores valores para magnitude da CIT somente no segundo momento do período competitivo (PCII) quando comparado aos demais momentos de competiçáo. Esse comportamento indica que a CIT aplicada no período preparatório e na maior parte do período competitivo foram similares. Freitas, Miloski e Bara-Filho ${ }^{2}$ descreveram a distribuição da CIT durante 5 mesociclos que antecederam a competição principal de jogadores de voleibol adultos e reportaram um aumento na magnitude dessa variável somente do primeiro para o segundo ciclo. Por outro lado, Aoki et al. ${ }^{3}$ apresentaram maiores valores de CIT durante o período de preparação comparado ao período competitivo, em jovens jogadores de voleibol brasileiros de elite, nas categorias sub16 e sub19.Maiores valores de CIT no período preparatório (vs. período competitivo) também foram reportados em jogadores de futsal ${ }^{11-12}$, futebol australiano ${ }^{17}$ e futebo ${ }^{19}$. Esses valores mais elevados de CIT no período preparatório tem sido utilizado para o incremento da aptidão física visando a etapa competitiva. Assim, a aplicação de CIT com valores similares entre o período preparatório e a maior parte do competitivo observada no presente estudo, difere das investigaçóes realizadas em outras modalidades de esporte coletivo, e pode ser explicada, possivelmente, pelas exigências específicas do calendário de cada equipe. Em geral, observa-se que os valores de CIT dos atletas de voleibol foram ligeiramente inferiores ao observado em jogadores futsal (acima de 4000 UA) e futebol profissionais (próximo de 4000 UA) durante o período preparatório. No entanto, devese registrar que a equipe investigada no presente estudo participou do campeonato estadual (como competição preparatória) durante as seis últimas semanas do período preparatório, contribuindo para a realização de um grande número de jogos. Embora essa competição tenha apresentado caráter preparatório, deve-se considerar a importância em se buscar um equilíbrio entre a demanda de estresse (proveniente de treinamentos, jogos e viagens) e os períodos de recuperação, para se evitar adaptaçóes indesejadas (ex. overreaching não-funcional, lesôes, infecçôes de trato respiratório superior) ${ }^{18-19}$. Nesse caso, a busca por esse equilíbrio parece ter impossibilitado a aplicação de semanas sucessivas com CIT elevada, e consequentemente, valores mais altos de CIT no período preparatório.

Mais um resultado interessante consiste na redução da CIT direcionada ao desenvolvimento da aptidáo física ( $\mathrm{F}$ e C+TT) durante o segundo momento do período competitivo. Outros estudos também mostraram uma redução da CIT direcionada ao desenvolvimento da aptidão física em jogadores profissionais de futsal ${ }^{11-12}$, futebol australiano ${ }^{17}$, em jovens jogadores de voleibol de elite $^{20}$. Em conjunto, esses resultados apontam que a redução da carga de treinamento direcionada ao desenvolvimento da aptidão física é uma estratégia comumente utilizada durante alguns momentos do período competitivo. Algumas investigaçóes realizadas em esportes coletivos têm apontado que a utilização dessa estratégia não compromete o nível de aptidáo física durante o período competitivo ${ }^{11}$. 
Em contrapartida, pode-se observar um aumento da magnitude da carga dos treinamentos que buscavam reproduzir o formato de jogo e desenvolver os aspectos técnico-táticos no período competitivo em relação ao preparatório, ao passo que a carga direcionada a treinamentos técnicos isolados se manteve constante no decorrer da temporada. Moreira et al. ${ }^{17}$, ao contrário, demonstraram redução da CIT direcionada as sessões específicas de futebol em jogadores profissionais de futebol australiano durante o período competitivo. Essa diferença observada para o comportamento da CIT TT no presente estudo, pode ser estar associada possivelmente ao calendário competitivo da equipe de voleibol estudada, devendo-se considerar que o intervalo entre os jogos pode ter possibilitado a aplicação de uma maior magnitude de CIT TT no período de competiçóes. Adicionalmente, os resultados também apontaram que durante o período competitivo houve uma ênfase na CIT aplicada nos treinamentos técnico-táticos. Esses resultados corroboram os achados de investigaçóes realizadas em jogadores de futsal profissionais ${ }^{11}$ e atletas de futebol australiano profissional ${ }^{17}$, e apontam para a preocupação das comissóes técnicas em relação ao aprimoramento do comportamento técnico-tático adotado em situaçóes de jogo, nas sessóes de treinamento realizadas entre os jogos nos esportes coletivos.

Os resultados do presente estudo são de grande relevância, uma vez que representam mais um passo em ralação ao estudo de Freitas, Miloski e BaraFILHo $^{12}$, ao descrever a distribuição da CIT em jogadores profissionais de voleibol, considerando não somente sua magnitude, como também seu direcionamento (força, jogos, técnico, técnicotático, condicionante e técnico-tático). Diversos estudos apontaram para a tendência de aplicação de CIT elevada no período preparatório e redução da mesma durante o período competitivo, em diferentes esportes coletivos ${ }^{11-12,17}$. O presente estudo, porém, demonstrou valores de CIT similares entre períodos preparatório e competitivo. Entretanto, os resultados desse estudo adicionam a informação de que ocorre uma redução da CIT direcionada ao desenvolvimento da aptidão física quando a CIT total é reduzida no período competitivo; e ainda, uma tendência de aumento da CIT direcionada aos atributos técnico-táticos em jogadores de voleibol de elite durante o período competitivo (vs. preparatório). Dessa maneira, a organização do treinamento descrita pode representar mais uma possibilidade de orientação para os profissionais do esporte. Entretanto, deve-se considerar que a descrição da distribuição da CIT de apenas uma única equipe representa uma limitação desse estudo. Assim, é possível que esta seja a distribuição de CIT de apenas uma equipe, e que, a organização da CIT durante uma temporada pode variar em diferentes equipes.

A partir dos resultados do presente estudo podese concluir que a carga interna de treinamento (CIT) apresentou uma distribuição que alternou diferentes magnitudes durante semanas de treinamento na temporada, tanto no período preparatório como no competitivo, como forma de se atender um equilíbrio adequado entre estímulos de estresse e recuperação nos jogadores de voleibol profissionais analisados. Além da alternância na magnitude da CIT semanal, nota-se que ocorre uma ênfase na CIT direcionada aos atributos técnico-táticos e redução da CIT destinada ao desenvolvimento da aptidão física durante o período competitivo da temporada do voleibol. Dessa maneira, o presente estudo aponta para novas possibilidades de monitoramento da CIT na prática diária das comissóes técnicas, considerando os diferentes tipos de treinamento separadamente, e ainda apresenta orientaçóes a distribuição das cargas de treinamento no voleibol profissional, considerando sua magnitude e seus conteúdos de treinamento. 


\section{Abstract \\ Internal training load distribution during a volleyball professional season}

This study aimed to describe the internal load distribution (ITL) related to different types of training during a season of Professional volleyball. ITL was registered in all training sessions during 30 weeks, taking into account following types of training: strength $(S)$, technical $(T)$, technical-tactical $(T)$, match $(M)$ e conditioning + technical-tactical $(C+\pi)$. The season was divided into 4 periods: preparatory (PP) - 8 weeks, competitive I (PCI) - 6 weeks, competitive II (PCII) - 7 weeks, and competitive III (PCIII) - 9 weeks. Rating of perceived exertion session method to quantify the ITL. The results show an alternation in ITL magnitude during the weeks in the season; a reduction of ITL F from PCI to PCII $(p=0,04)$; greater values of CIT TT from $\mathrm{PCl}$ and PCIII compared to PP $(p<0,01)$; and lower values to $C+\Pi$ at $P C I I$ compared to other periods $(p<0,01)$. It can be concluded that ITL showed a distribution with alternation in their magnitude among of season, an emphasis in ITL to technical-tactical atributes and a decrease in ITL to develop physical fitness during competitive period in a volleyball season.

KEYwoRDS: RPE-session; Periodization; Monitoring; Training load.

\section{Referências}

1. Issurin VB. New Horizons for the Methodology and Physiology of Training Periodization. Sports Med. 2010;40(3):189206.

2. Freitas VH, Miloski B, Bara Filho MG. Monitoramento da carga interna de um período de treinamento em jogadores de voleibol. Rev Bras Educ Fis Esporte. 2015;29(1):5-12.

3. Aoki MS, Torres-Ronda L, Marcelino PR, Drago G, Carling C, Bradeley P, Moreira A. Monitoring training loads in professional basketball players engaged in a periodized training programme. J Strength Cond Res. 2016; 31:348-358.

4. Impellizzeri FM, Rampinini E, Coutts AJ, Sassi A, Marcora SM. Use of RPE-based training load in soccer. Med Sci Sports Exerc. 2004;36(6):1042-1047.

5. Foster C, Florhaug JA, Franklin J, Gottschall L, Hrovatin LA, Parker S, et al. A new approach to monitoring exercise training. J Strength Cond Res. 2001;15(1):109-115.

6. Lovell TWJ, Sirotic AC, Impellizzeri FM, Coutts AJ. Factors affecting perception of effort (session rating of perceived exertion) during Rugby League training. Int J Sports Physiol Performance. 2013;8(1):62-69.

7. Franchini E, Tabben M, Chaabène H. Physiological responses during Taekwondo training and competition. Int Sport Med J FIMS. 2014; 15:500-515.

8. Bara Filho MG, Andrade FCd, Nogueira RA, Nakamura FY. Comparação de diferentes métodos de controle da carga interna em jogadores de voleibol. Rev Bras Med Esporte. 2013;19(2):143-6.

9. Algroy EA, Hetlelid KJ, Seiler S, Pedersen JIS. Quantifyng training intensity distribuition in a group of norwegian professional soccer players. Int J Sports Physiol Performance. 2011; 6:70-81.

10. Foster C, Heimann K, Esten P, Brice G, Porcari J. Differences in perceptions of training by coaches and athletes. S Afr J Sports Med. 2001;8(2):3-7.

11. Miloski B, Freitas VH, Nakamura FY, De ANFC, Bara-Filho MG. Seasonal training load distribution of professional futsal players: effects on physical fitness, muscle damage and hormonal Status. J Strength Cond Res. 2016;30(6):1525-1533. 12. Miloski B, Freitas VHd, Bara Filho MG. Monitoring of the internal training load in futsal players over a season. Rev Bras Cineantropom Desempenho Hum. 2012;14(6):671-679.

13. Moreira A, Freitas CG, Nakamura FY, Drago G, Drago M, Aoki MS. Eff ect of match importance on salivary cortisol and immunoglobulin a responses in elite young volleyball players. J Strength Cond Res. 2013; 27(1):202-207.

14. Moreira A, McGuigan M R, Arruda AFS, Freitas CG, Aoki MS. Monitoring internal load parameters during simulated and official Basketball Matches. J Strength Cond Res. 2012; 26(3):861-866.

15. Skein M, Duffi eld R, Minett GM, Snape A, Murphy A. The effect of overnight sleep deprivation after competitive Rugby League matches on postmatch physiological and perceptual recovery. Int J Sports Physiol Performance. 2013;8: 556-564. 
16. Kelly VG, Coutts, AJ. Planning and monitoring training loads during the competition phase in team sports. Strength Cond J. 2007; 29(4): 32-37.

17. Moreira A, Bilsborough JC, Sullivan CJ, Ciancosi M, Aoki MS, Coutts AJ. The training periodization of professional Australian Football players during an entire AFL season. Int J Sports Physiol Performance. 2015; 10:566-571.

18. Borresen J, Lambert MI. Th e quantifi cation of training load, the training response and the eff ect on performance. Sports Med. 2009;39(9):779-795.

19. Meeusen R, Duclos M, Foster C, Fry A, Gleeson M, Nieman D, Raglin J, Rietiens G, Steinacker J, Urhausen A. Prevention, diagnosis and treatment of the overtraining syndrome: joint consensus statement of the European College of Sport Science (ECSS) and the American College of Sports Medicine (ACSM). Eur J Sport Sci. 2013; 13:186-205.

20. Aoki MS, Arruda AFS, Freitas CG, Miloski B, Marcelino PR, Drago G, Drago M, Moreira A. Monitoring training loads, mood states, and jump performance over two periodized training mesocycles in elite young volleyball players [Internet]. Int J Sports Sci Coach. 2017;12: 130-137.

\begin{tabular}{r|r} 
ENDEREÇO & \\
Heglison Custódio Toledo & Submetido: 19/07/2017 \\
Universidade Federal de Juiz de Fora & 1ª Revisão: 20/11/2017 \\
Rua J osé Lourenço Kelmer, s/n - Campus Universitário & 2a Revisão: 23/02/2018 \\
36036-900 - Juiz de Fora - MG - Brasil & Aceito: 29/03/2018 \\
E-mail: hctoledo2@gmail.com & \\
& \\
\hline
\end{tabular}

